\title{
Frequency and Time Response of Power Plant Grounding System Exposed to Lightning Strike
}

\author{
Srete N. Nikolovski, Zoran Baus, Goran Knežević
}

Department of Power Engineering, Faculty of Electical Engineering, Josip Juraj Strossmyer University of Osijek, Croatia

\begin{tabular}{l}
\hline \hline Article Info \\
\hline Article history: \\
Received Nov 6, 2015 \\
Revised Dec 19, 2015 \\
Accepted Jan 4, 2016 \\
\hline Keyword: \\
Electromagnetic field \\
Field theory \\
Frequency spectrum \\
Grounding system \\
Lightning current \\
Scalar potential \\
Transients \\
\hline
\end{tabular}

\begin{abstract}
This paper examines the frequency response of power plant grounding system exposed to the lightning current. Large amount of current generated by the stroke flow in the grounding system of power plant and dissipate in the soil. The electric and magnetic field generated by such high voltages and currents may cause damage of equipment and may be dangerous for the personnel in power plant. For the every given frequency obtained using Fast Fourier Transformation (FFT) of lightning current impulse, electromagnetic field theory approach is used to solve Maxell's equation and compute scalar potential, electric and magnetic field. Also, the influence of the point in which lightning current is diffused in the grounding system is presented. Three dimensional plots of spatial distribution of scalar potential, electric and magnetic field are presented. The time domain response of scalar potential, electric and magnetic field on one profile is also presented.
\end{abstract}

Copyright (C) 2016 Institute of Advanced Engineering and Science. All rights reserved.

\section{Corresponding Author:}

Srete Nikolovski,

Departement of Power Engineering,

Faculty of Electical Engineering, Josip Juraj Strossmyer University of Osijek

K. Trpimira 2B, 31000 Osijek, Croatia.

Email:1srete.nikolovski@etfos.hr

\section{INTRODUCTION}

The transient behavior of thermal power plant grounding system buried in soil, with good resistivity subjected to the lighting current is examined using field theory approach. Different approach are used to solve the problem of generated EMF during transients caused by lightning strike to metallic parts of power plants, transformer stations, transmission line towers, buildings and communications towers. Recently computerized analysis methods had been developed using different approaches, for example circuit theory [12], transmission line theory [3-4], electromagnetic field theory [5-6], and hybrid theory [7-9].

In this paper electromagnetic field theory approach is used to solve Maxell's equation and compute scalar potential, electric and magnetic field. The technique used in the paper is based on the electric field point matching approach using Method of Moments (MoM).

In this paper the CDEGS- Current Distribution in Earth and Grounding System, software is used for modeling and simulation [10-11]. Two engineering modules from CDEGS are used, first HIFREQ and second FFTSES. With first module HIFREQ, GPR-Ground Potential Rise, scalar potential, electric and magnetic field are calculated for each frequency obtained by FFT- Fast Fourier Transformation of input lightning current impulse. Time domain response of EMF is then obtained by help of Inverse FFT of calculated GPR, scalar potential, electric and magnetic field at each frequency. HIFREQ module calculates current distribution for a network consisting aboveground and underground conductors excited by arbitrary frequencies. The network of conductors is subdivided in small segments. This enables the tin-wire approximation to be used with linear current sources. [12-13] 


\section{MATHEMATECAL MODEL}

From the well-known Maxwell's equations, it is possible to determinate the electric field $\vec{E}$ and the magnetic field $\vec{H}$ from the scalar potential $\varphi$ and the vector potential $\vec{A}$. The scalar potential, the electric field and the magnetic field can be express by equation given in [14]

$$
\begin{aligned}
& \varphi=\frac{1}{-\mu \vartheta} \nabla \cdot \vec{A} \\
& \vec{E}=-j \omega \vec{A}+\nabla \varphi \\
& \vec{H}=\frac{1}{\mu} \cdot \nabla \times \vec{A}
\end{aligned}
$$

Where are:

$$
\begin{aligned}
& \vec{A}-\text { Hertz vector potential } \\
& \vartheta=\sigma+j \omega \varepsilon-\text { complex conductivity of the soil } \\
& \mu-\text { permeability of the medium } \\
& \sigma-\text { conductivity of the medium } \\
& \varepsilon-\text { permittivity of the medium. }
\end{aligned}
$$

Different models for lightning current waveforms can be considered; standard exponential function, double exponential function and Heidler function [13]. The lightning waveform is usually defined by the rise time $t_{f}$, pulse length $t_{p}$ and peak value $I$. In order to represent lightning current waveforms in an analytic manner, the double-exponential formula is employed to fit with the lightning current waveforms. Equation is given in expression (4).

$$
I(t)=I_{m}\left(e^{-a \cdot t}-e^{-b \cdot t}\right)
$$

In our case most common values for lightning current peak value are $I_{m}=10(20,40,100) \mathrm{kA}$, coefficient $a=1,386 \times 10^{4} \mathrm{~s}^{-1}$, coefficient $b=6 \times 10^{6} \mathrm{~s}^{-1}$ The magnitude of lightning current $I_{m}=10 \mathrm{kA}$ in simulation is used. With these coefficients $a$ and $b$ the waveform of lightning current impulse is characterized by a rising time of $1,2 \mu \mathrm{s}$, and half-value time of $50 \mu \mathrm{s}$ which is typical testing waveform.

\section{CASE STUDY}

Grounding system of power plant is asymmetrical and complex buried structure which consists of $\mathrm{Fe} / \mathrm{Zn} 30 \times 5 \mathrm{~mm}^{2}$ conductors and grounding rods. Conductors form asymmetrical grounding grid buried at the depth of $0,46 \mathrm{~m}$. The equivalent diameter of the grounding conductors is $\mathrm{d}=0,0124 \mathrm{~m}$ (for Fe/ $\mathrm{Zn} 30 \times 5 \mathrm{~mm}^{2}$ ). Grounding system also has twelve $3.5 \mathrm{~m}$ grounding rods, buried from $0,46 \mathrm{~m}$ in the soil. Grounding system is shown in Figure 1.

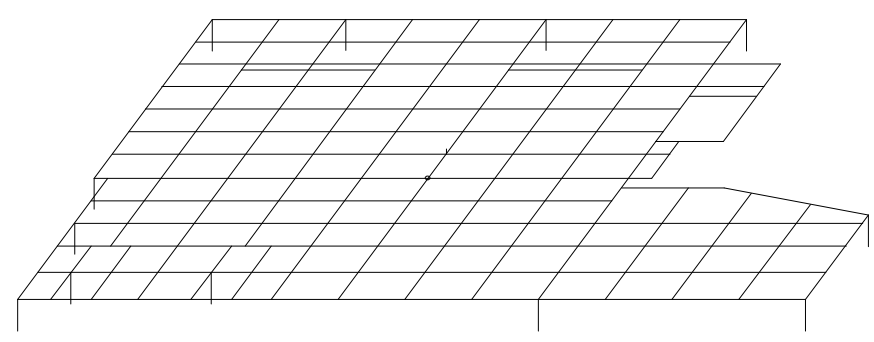

Figure 1. The asymmetrical grounding grid with grounding rods

An observation surface is on the level 0 on the ground on which scalar potential, electric field and magnetic field where calculate consists of 70 profiles on distance of $1 \mathrm{~m}$. Each profile is divided on 80 observation points separate one from another on distance of $1 \mathrm{~m}$. The total number of observation points is 
5600. Two layer soil models is used with soil parameters. Top layer soil resistivity is $100 \Omega \mathrm{m}$. Top layer soil depth $10 \mathrm{~m}$. Bottom layer soil resistivity $10 \Omega \mathrm{m}$. Bottom layer depth is infinity. Relative permittivity and permeability ob the both layers are $1 \mathrm{pu}$. The first case examined in the paper is when that lightning current impulse hits in the middle of the grounding grid. The second case examined in the paper is when that lightning current impulse hits in left corner of the grounding grid.

\subsection{Scalar Potential for Different Frequencies, $1^{\text {st }}$ Case}

When lightning current waveform is transferred in the frequency domain, by use of FFT transformation only fourteen frequencies are recommended for calculation of $\varphi, E$ and $H$. [14]. Do thelack of the space in the paper only three representative frequencies are chosen: $50 \mathrm{~Hz}, 2 \mathrm{kHz}$ and 1,68 MHz. For frequency of $50 \mathrm{~Hz}$ maximum of induced scalar potential at the ground level is 7,98 kV. Figures $3-4$ present spatial distribution of scalar potential for the frequencies of $2 \mathrm{kHz}$ and 1,68 MHz. For frequency of $2 \mathrm{kHz}$ maximum induced scalar potential at the ground level is $9,43 \mathrm{kV}$, Figure 3. For frequency of $1,68 \mathrm{MHz}$ maximum of induced scalar potential at the ground level is $92,67 \mathrm{kV}$.

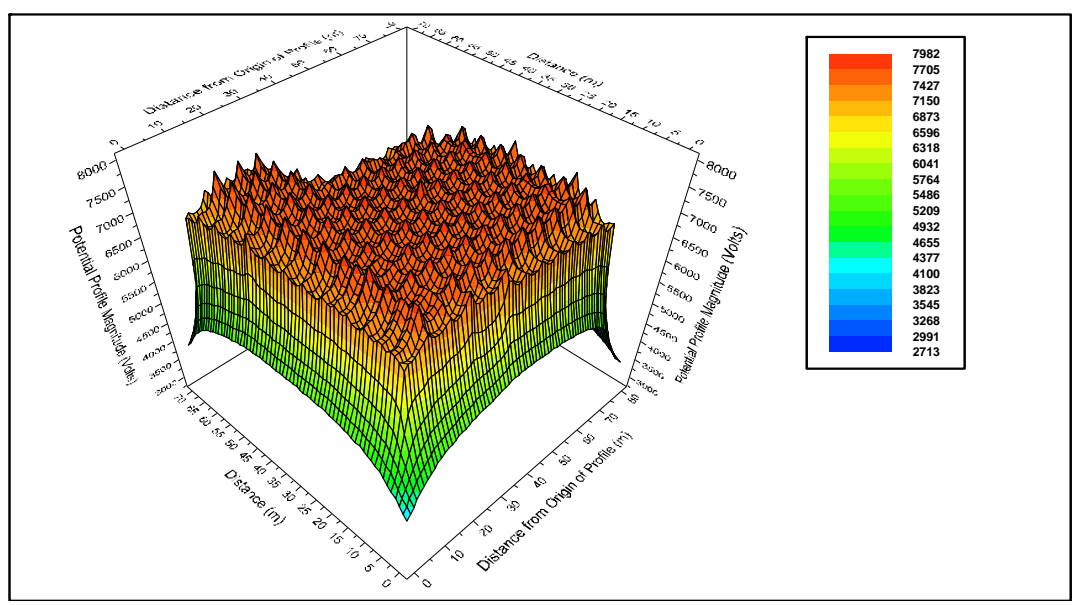

Figure 2. The spatial distribution of scalar potential at the ground level for the frequency $50 \mathrm{~Hz}$

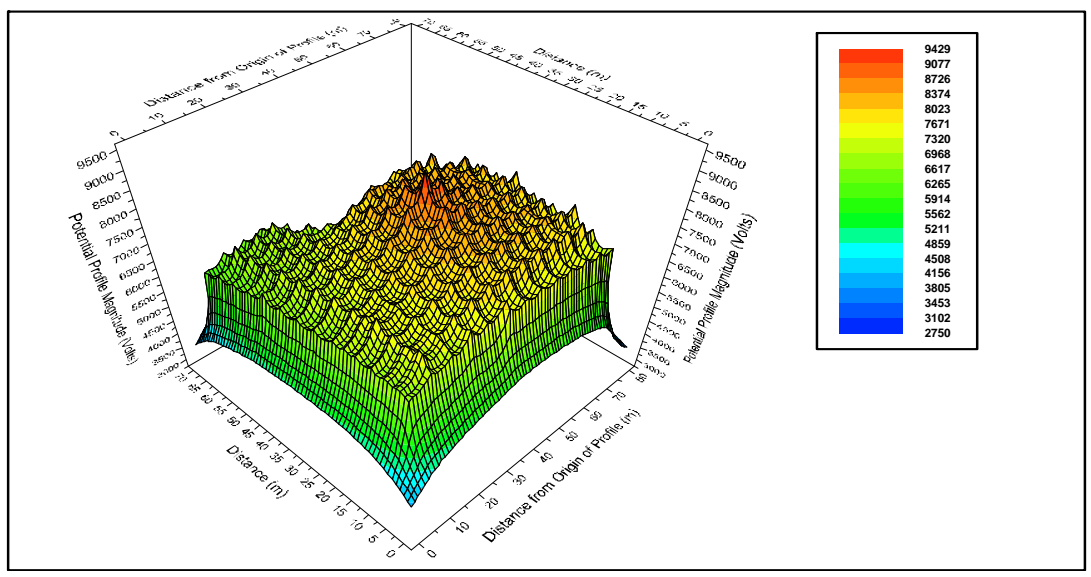

Figure 3. The spatial distribution of scalar potential at the ground level for the frequency of $2 \mathrm{kHz}$ 


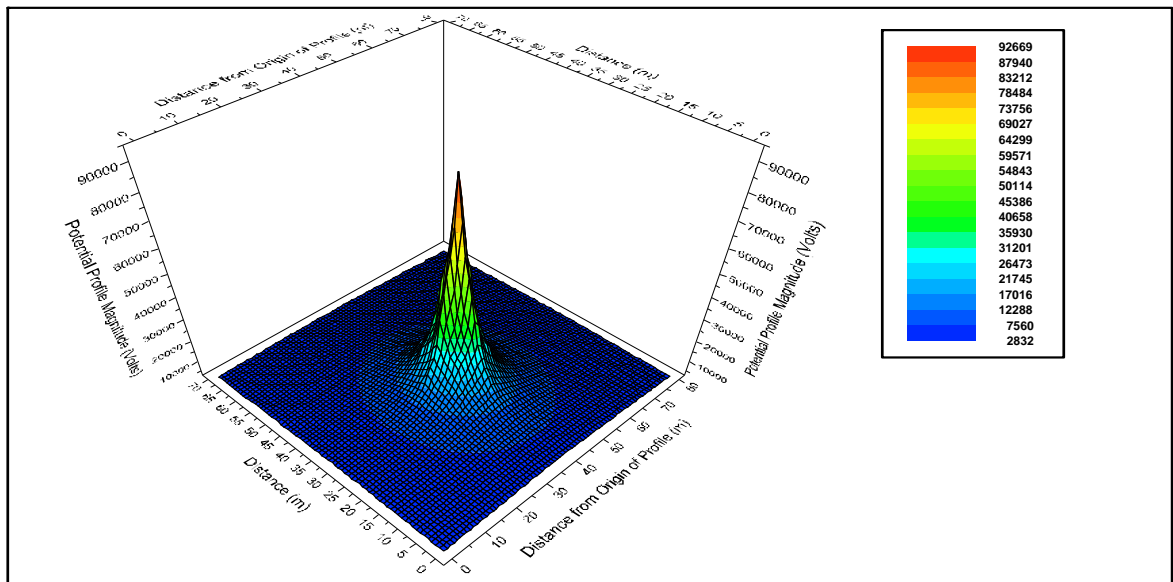

Figure 4. The spatial distribution of scalar potential at the ground level for frequency $1,68 \mathrm{MHz}$

\subsection{Electric Field for Different Frequencies, $1^{\text {st }}$ Case}

The spatial distributions of electric field for the same frequencies are shown in Figure 5 to Figure 7.

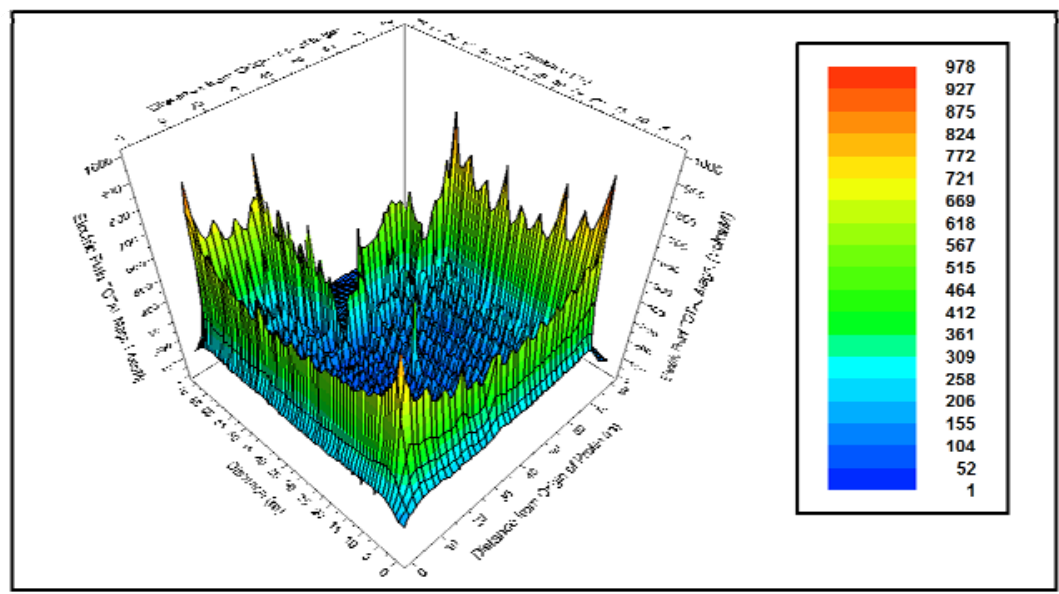

Figure 5. The spatial distribution of electric field at the ground level for the frequency of $50 \mathrm{~Hz}$

The maximum of the electric field is $978 \mathrm{~V} / \mathrm{m}$ at the point where current strike hits the object above grounding grid. For the frequency of $2 \mathrm{kHz}$ maximum of the electric field is $1119 \mathrm{~V} / \mathrm{m}$ 


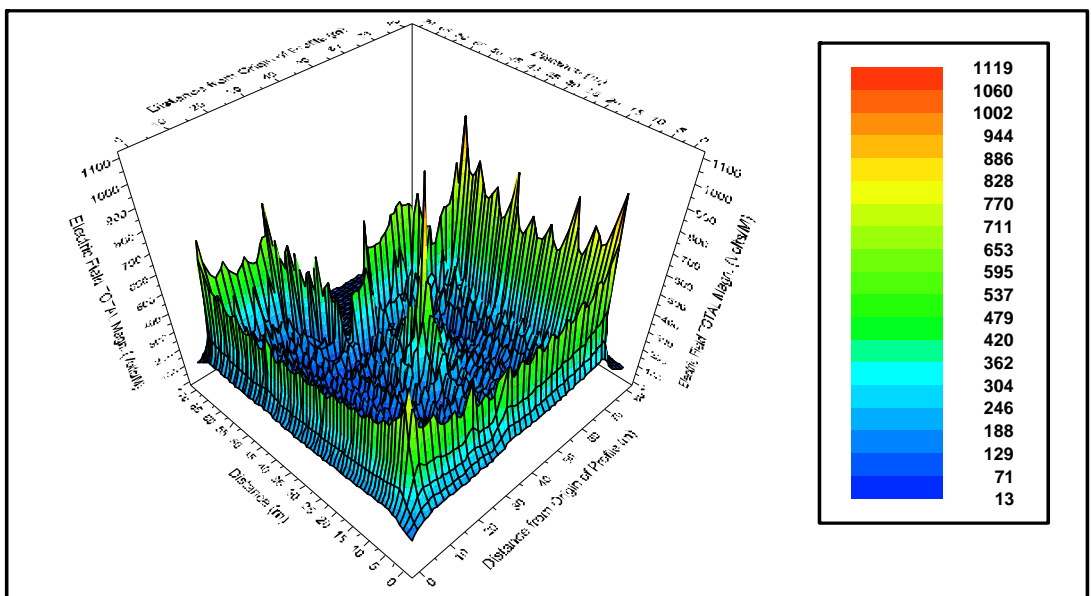

Figure 6. The spatial distribution of electric field at the ground level for the frequency of $2 \mathrm{kHz}$

For the frequency of $1,68 \mathrm{MHz}$ maximum of the electric field is $45176 \mathrm{~V} / \mathrm{m}$.

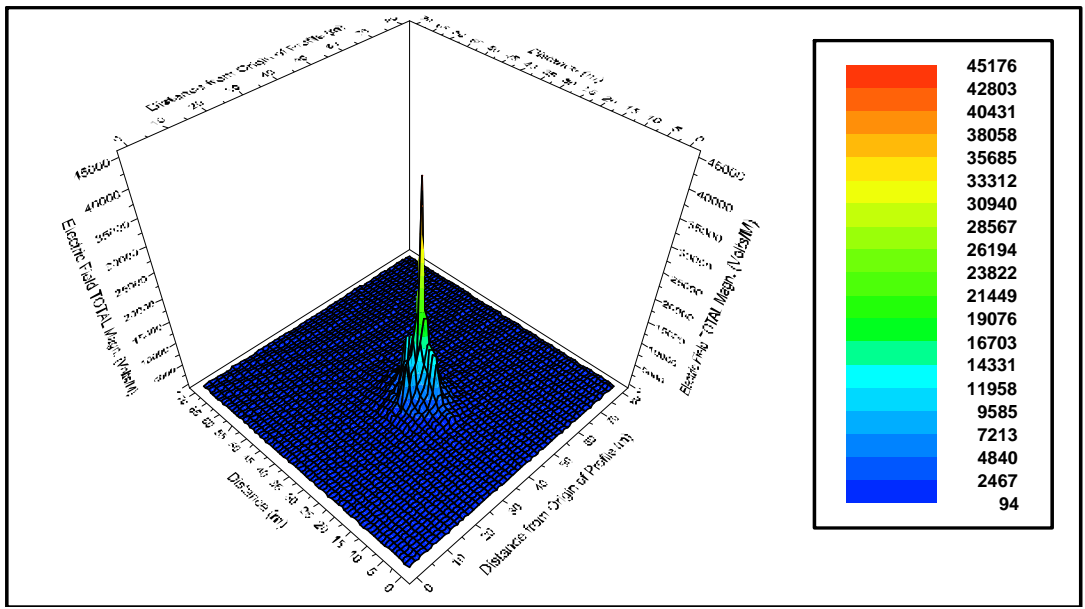

Figure 7. The spatial distribution of electric field at the ground level for frequency of $1,68 \mathrm{MHz}$

\subsection{Magnetic Field for Different Frequencies, $1^{\text {st }}$ Case}

The spatial distributions of magnetic field are shown only for frequencies of $50 \mathrm{~Hz}$ and $1,68 \mathrm{MHz}$ in Figure 8 and Figure 9. Magnetic file do not depend on the frequency changes. The maximum value is 1256 $\mathrm{A} / \mathrm{m}$. 


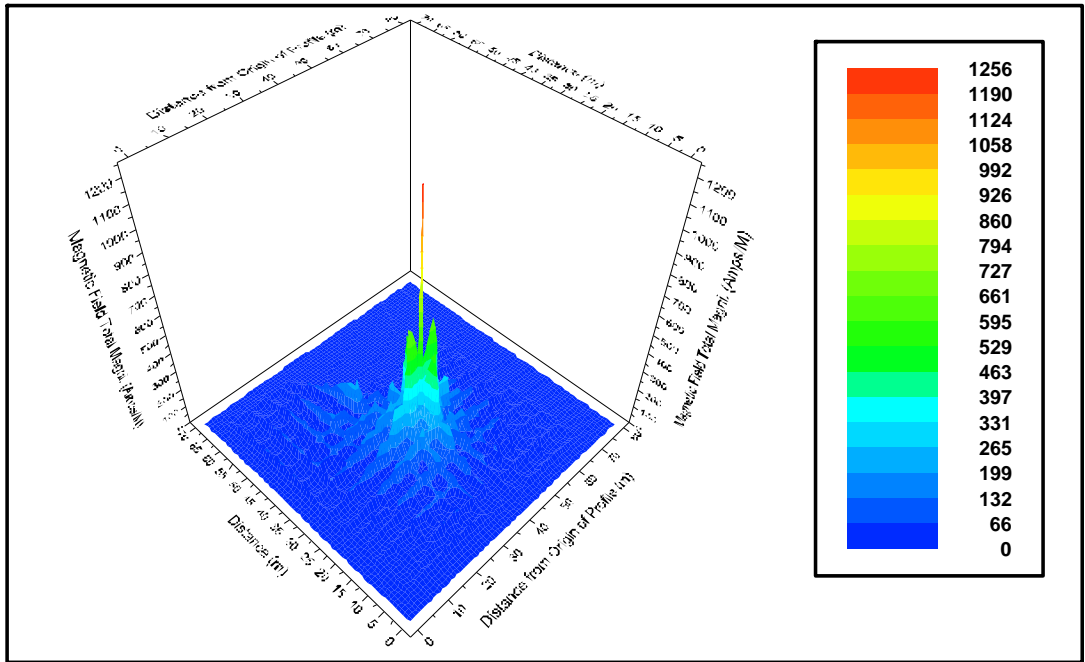

Figure 8. The spatial distribution of magnetic field at the ground level for the frequency of $50 \mathrm{~Hz}$

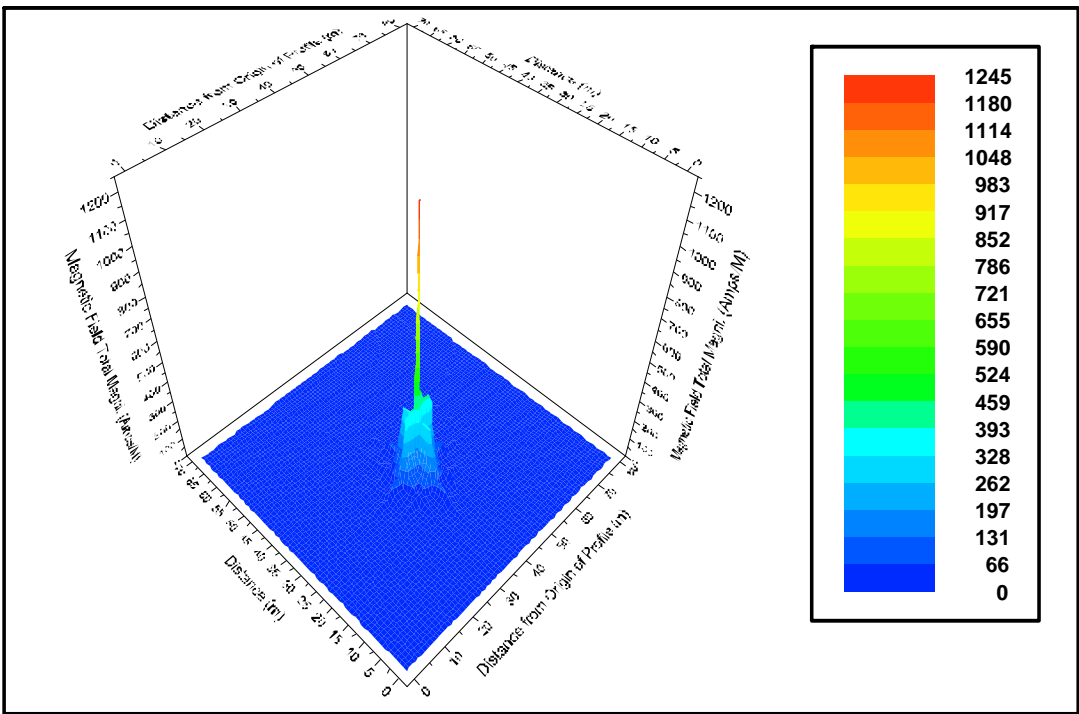

Figure 9. The spatial distribution of magnetic field at the ground level for frequency of 1,68 MHz

\subsection{Scalar Potential for Different Frequencies, $2^{\text {nd }}$ Case}

Here, also three representative frequencies are chosen: $50 \mathrm{~Hz}, 2 \mathrm{kHz}$ and 1,68 MHz. The spatial distributions of scalar potential are shown in Figure 10 to Figure 12.

For the frequency of $50 \mathrm{~Hz}, 2 \mathrm{kHz}$ and $1,68 \mathrm{MHz}$ maximum of induced scalar potentials at the ground level are $10,452 \mathrm{kV}, 25,77 \mathrm{kV}$, and $92,21 \mathrm{kV}$, respectively. 


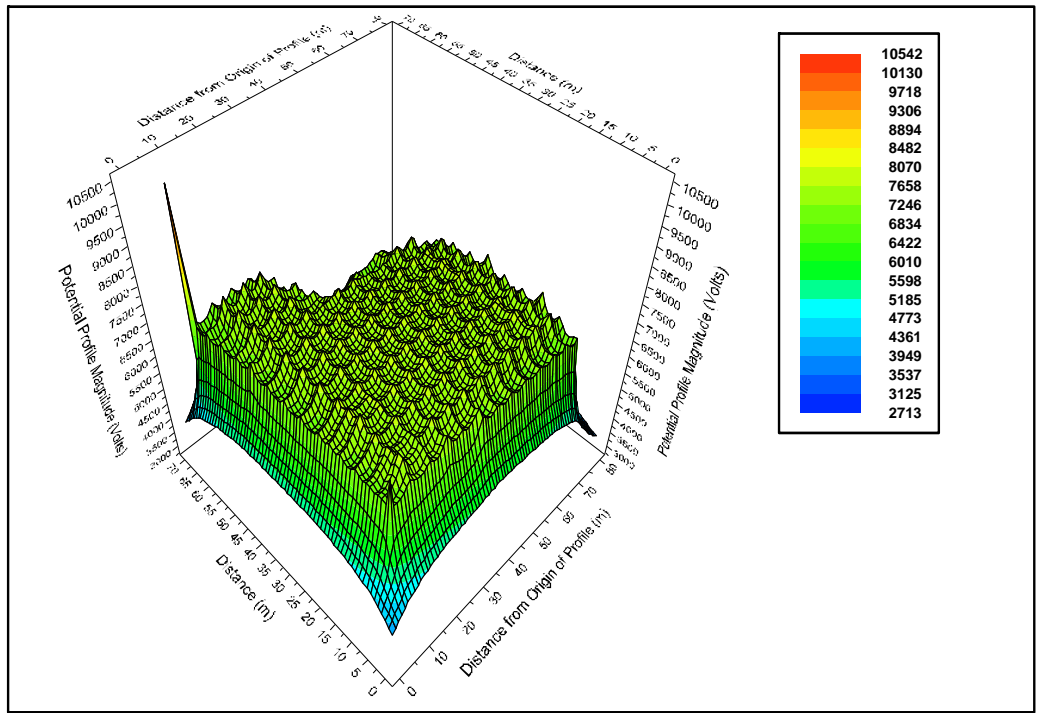

Figure 10. The spatial distribution of scalar potential at the ground level for frequency of $50 \mathrm{~Hz}$

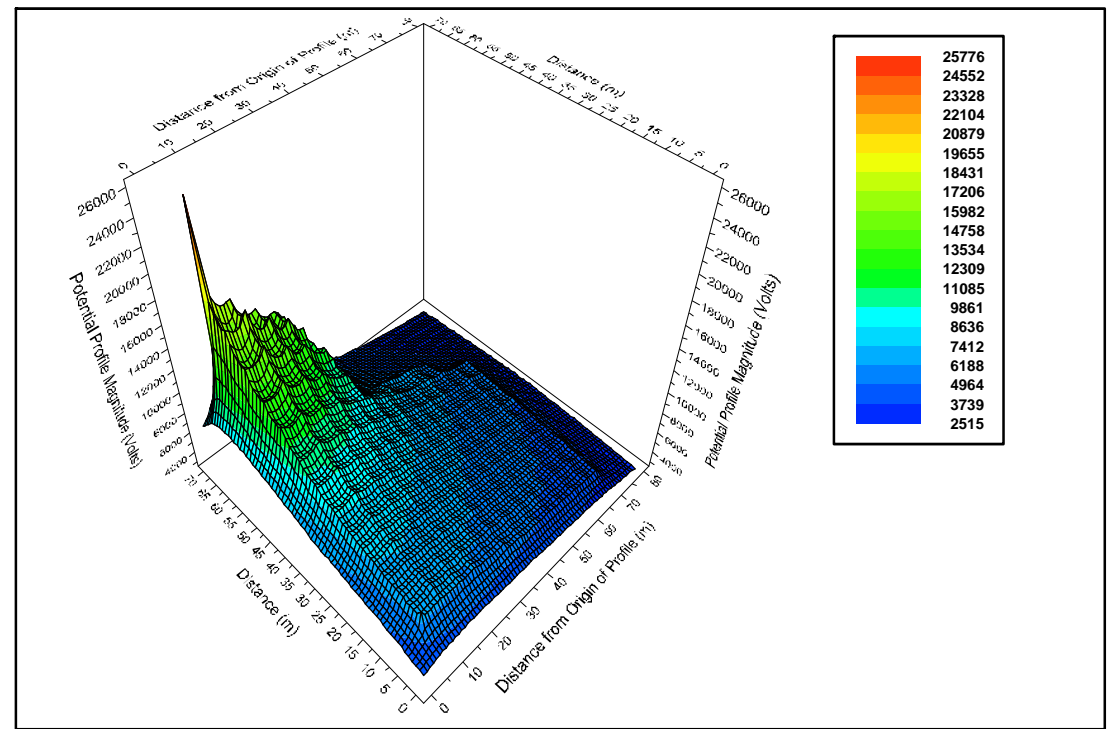

Figure 11. The spatial distribution of scalar potential at the ground level for frequency of $2 \mathrm{kHz}$ 


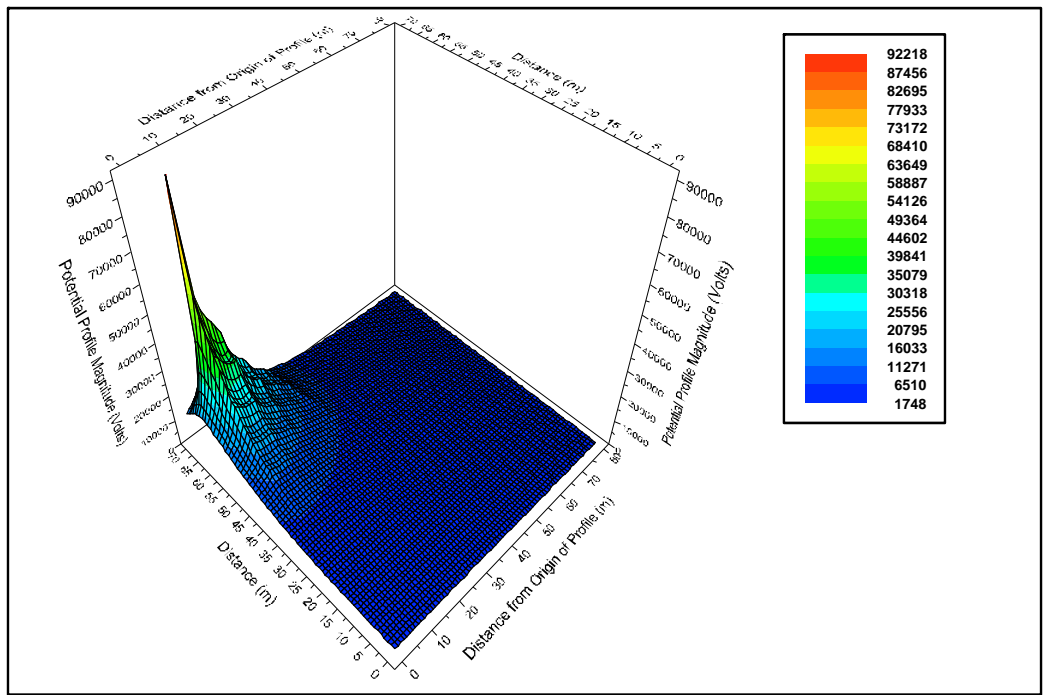

Figure 12. The spatial distribution of scalar potential at the ground level for frequency of 1,68 MHz

\subsection{Electric Field for Different Frequencies, $2^{\text {nd }}$ Case}

The spatial distributions of electric field for only three frequencies are shown in Figure 13 to Figure 15.

For the frequency of $50 \mathrm{~Hz}$ the maximum of induced electric field at the ground level is $978 \mathrm{~V} / \mathrm{m}$. The amount is the same as in the case when the lightning impulse hit the center of the grounding grid. For the frequency of $2 \mathrm{kHzand} 1,68 \mathrm{MHz}$ the maximum of induced electric field at the ground level are $4402 \mathrm{~V} / \mathrm{m}$ and $81871 \mathrm{~V} / \mathrm{m}$, respectively.

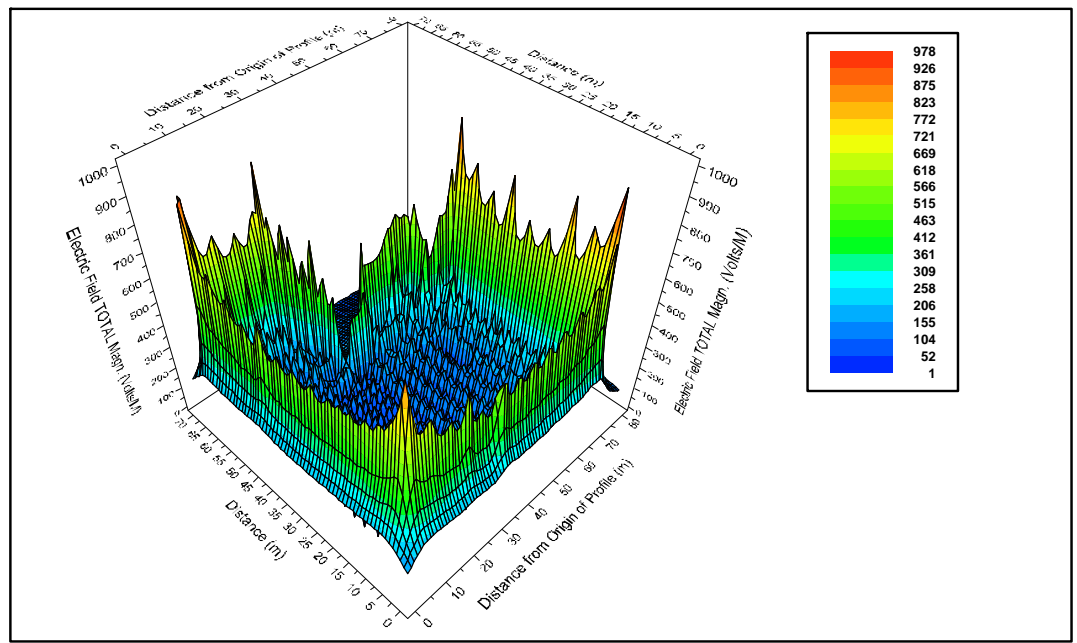

Figure 13. The spatial distribution of electric field at the ground level for frequency of $50 \mathrm{~Hz}$ 


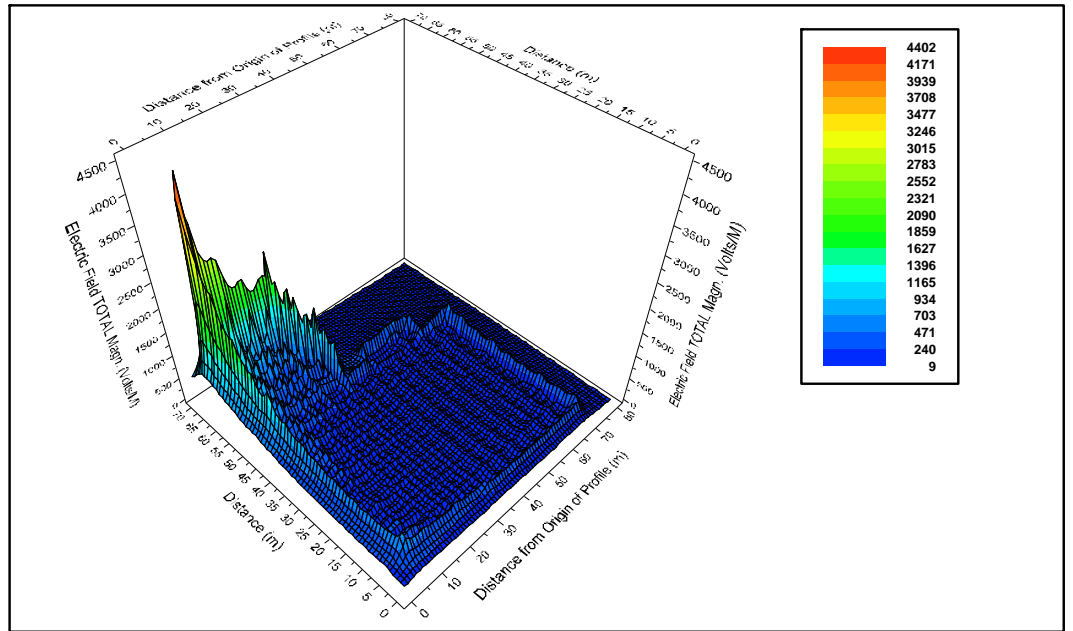

Figure 14. The spatial distribution of electric field at the ground level for frequency of $2 \mathrm{kHz}$

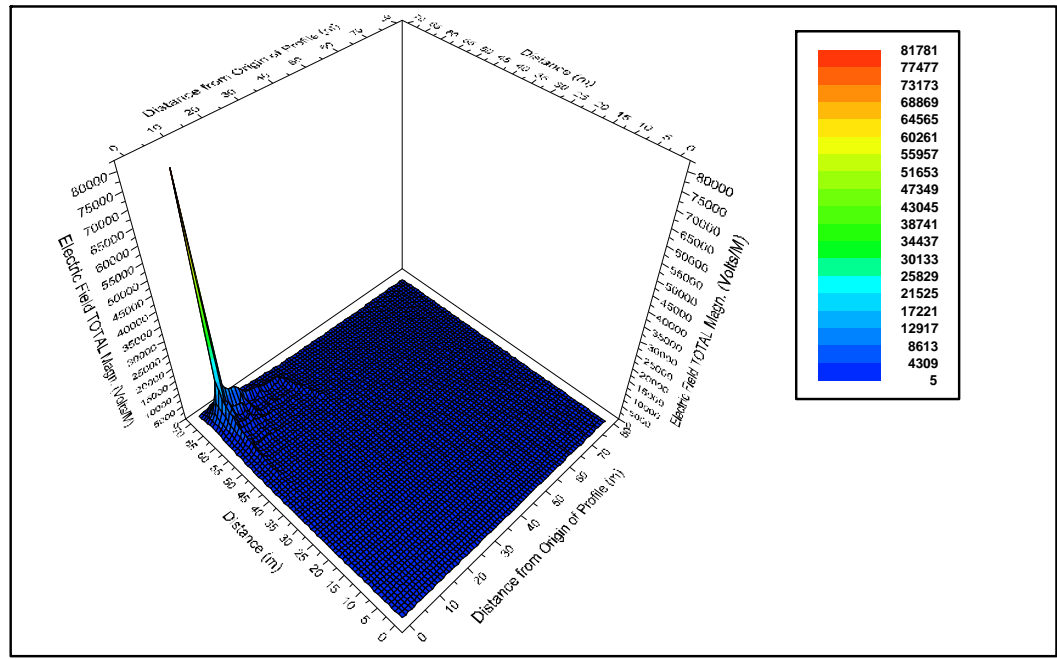

Figure 15. The spatial distribution of electric field at the ground level for frequency of $1,68 \mathrm{MHz}$

\subsection{Magnetic Field for Different Frequencies, $2^{\text {nd }}$ Case}

The spatial distributions of magnetic field for only three frequencies are shown in Figure 16 to Figure 18. Magnetic field does not depend on frequency changes. The spatial distribution of magnetic filed for $2 \mathrm{kHz}$ is the same as previeous case and is $1526 \mathrm{~A} / \mathrm{m}$. For the frequency of $1,68 \mathrm{MHz}$ the maximum of induced magnetic field at the ground level is $1236 \mathrm{~A} / \mathrm{m}$. 


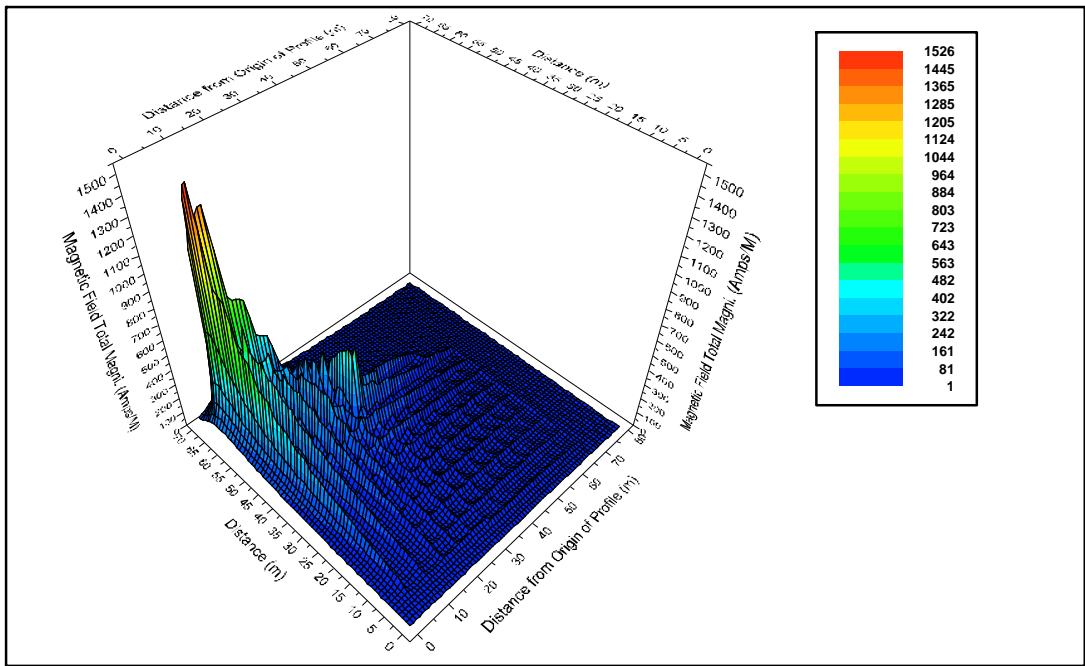

Figure 16. The spatial distribution of magnetic field at the ground level for frequency of $50 \mathrm{~Hz}$

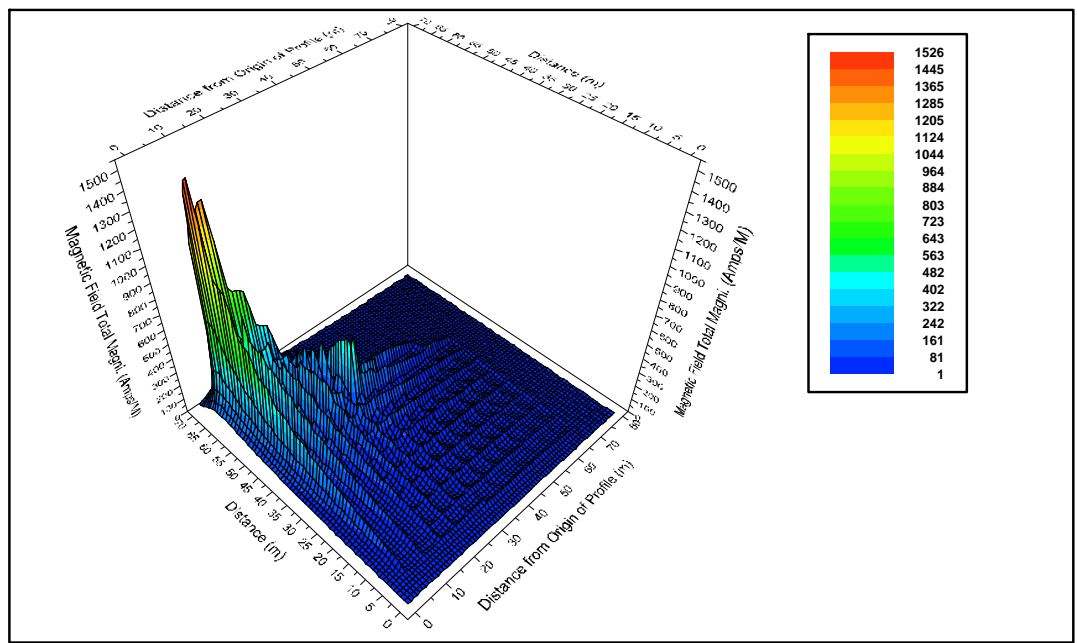

Figure 17. The spatial distribution of magnetic field at the ground level for frequency of $2 \mathrm{kHz}$

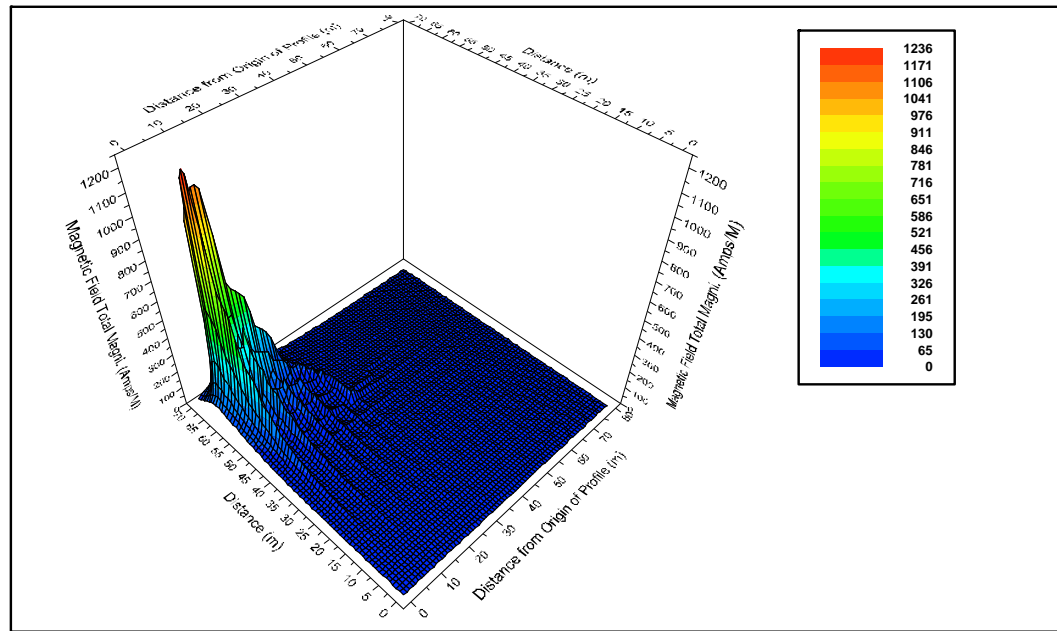

Figure 18. The spatial distribution of magnetic field at the ground level for frequency of 1,68 MHz 


\section{TIME DOMAIN ANALYSIS}

The time-domain evolution of the electromagnetic fields was obtained by first computing the skalar potential electric and magnetic fields in the frequency domain for a representative sample of 110 frequencies, Time-domain analyses are calculate using Inverse FFT with time span of $150 \mu$ s like in. [14]

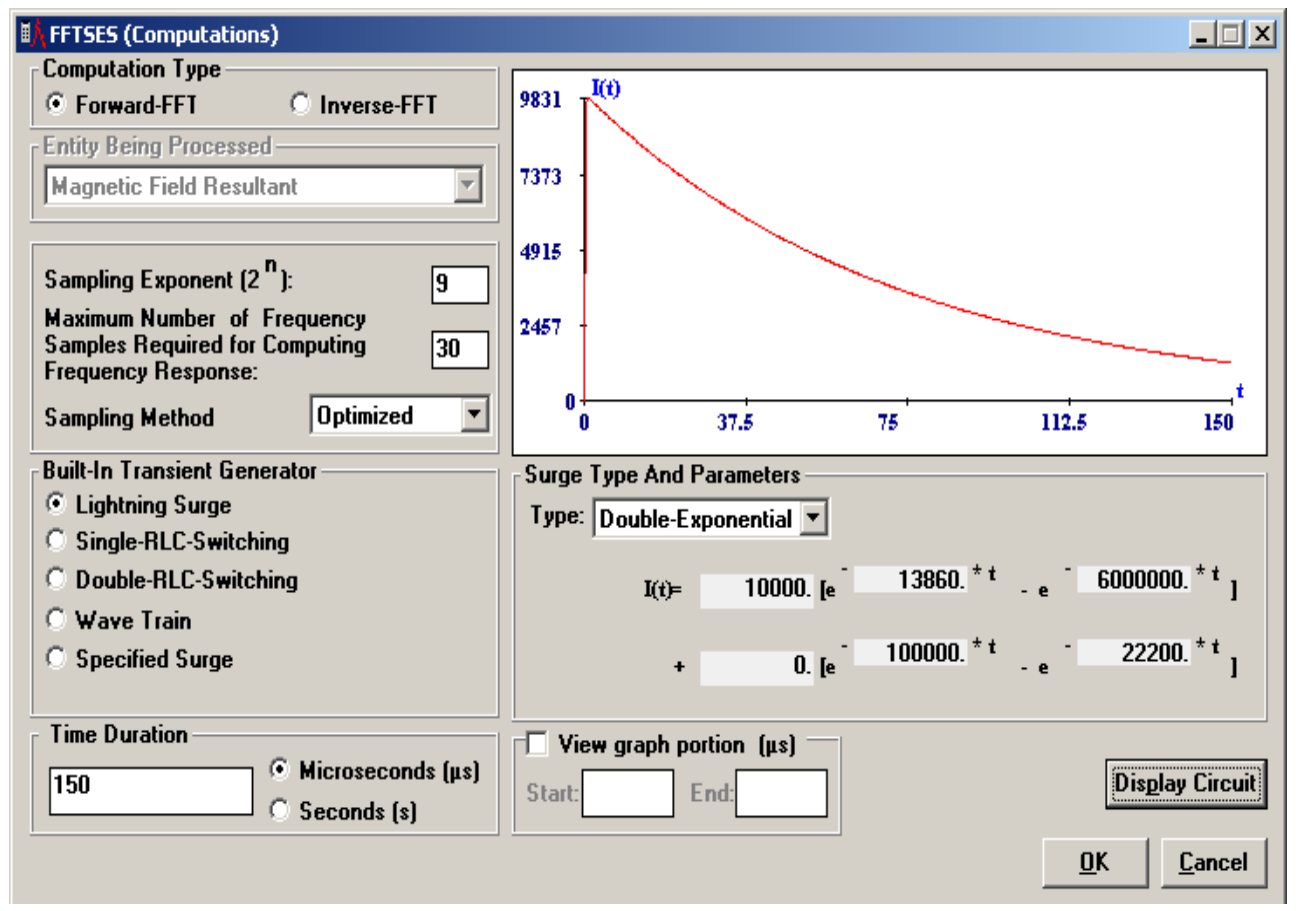

Figure 19. Lightning current waveform

The time evolution of scalar potential for the case when lightning impulse hit the center of the grounding grid is calculated across the $30^{\text {th }}$ profile at $80^{\text {th }}$ observation points is presented in Figure 20 to Figure 22. The time evolution of scalar potential for the case when lightning impulse hit the corner of the grounding grid is calculated across the $5^{\text {th }}$ profile at $80^{\text {th }}$ observation points is presented in Figure 23 to Figure 25 .

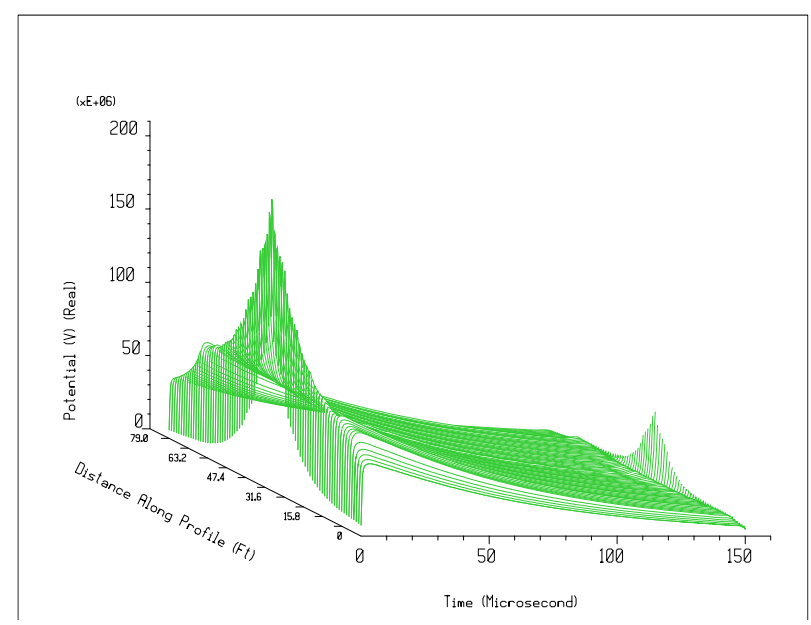

Figure 20. Time waveform of scalar potential across the 30th profile for the case one 


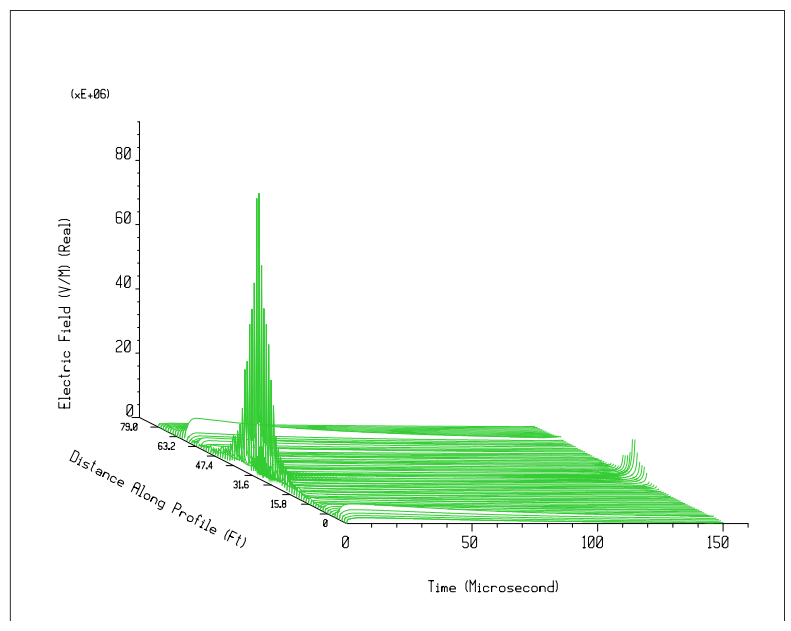

Figure 21. Time waveform of electric field across the 30th profile for the case one

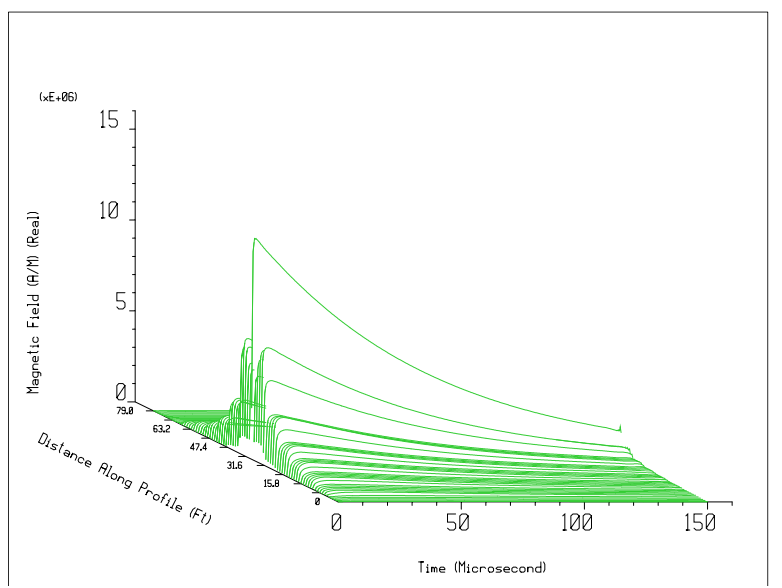

Figure 22. Time waveform of magnetic field the 30th profile for the case one

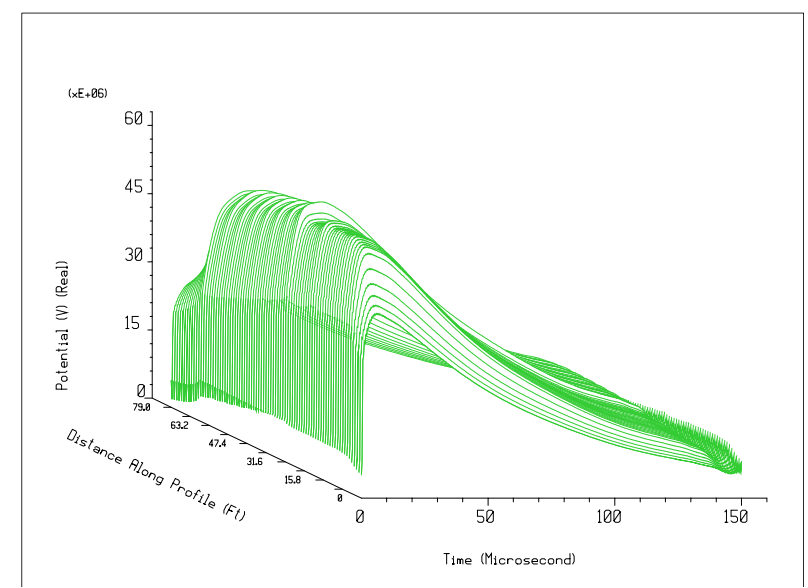

Figure 23. Time waveform of scalar potential at the 5 th profile for the case two 


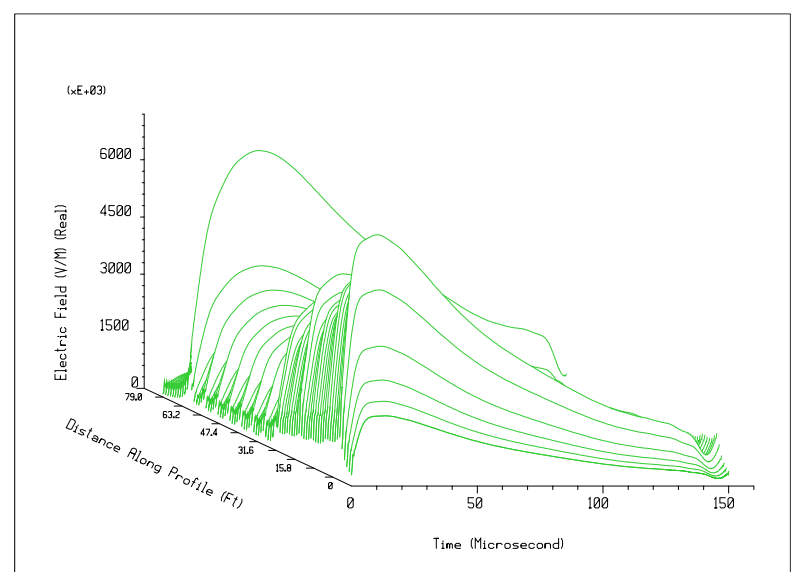

Figure 24. Time waveform of electric field at the 5th profile for the case two

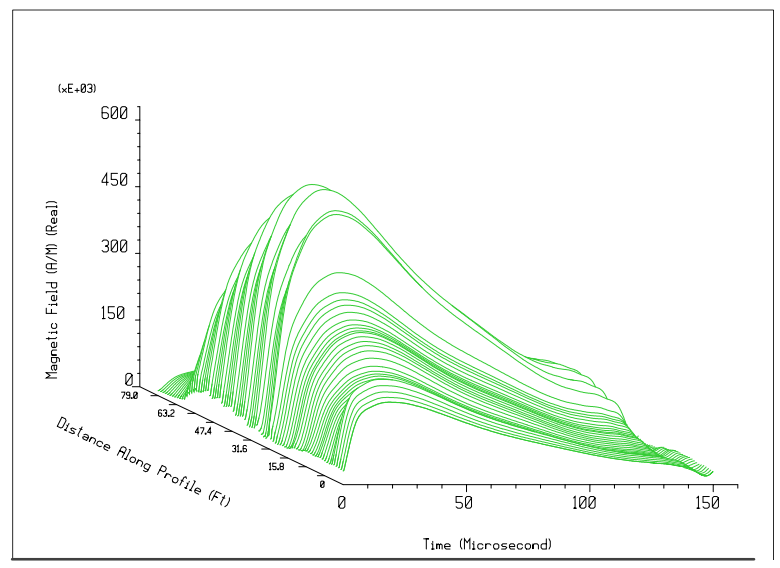

Figure 25. Time waveform of magnetic field at the 5th profile for the case two

All quantities scalar potential, electric and magnetic field in time domain are smaller in the second case when lightning impulse hit the corner of the grounding grid.

\section{CONCLUSION}

In this paper a detailed frequency response of power plant grounding system exposed to the lightning current impulse has been described and analysed. Earth scalar potential, electric and magnetic field at the ground level for different frequencies have been computed and plotted for two different scenarios. The results of this paper show significant influence of frequency values on the spatial distribution of the scalar potentials and the electric fields, while the magnetic fields is weakly dependent on frequency changes. In case of low frequencies, scalar potential and electric field are not affected by the location of the lightning current injection.

\section{ACKNOWLEDGEMENTS}

The authors gratefully acknowledge the contribution and technical support of SES technologies ltd,

\section{REFERENCES}

[1] E.D. Sunde. Earth Conduction Effects in Transmission Systems, New York: Dover, 1968.

[2] L.V. Bewley, Traveling Waves on Transmission Systems, 2nd ed. New York: Wiley, 1951.

[3] R. Rudenberg, Electrical ShockWaves in Power Systems. Harvard Univ. Press, 1968.

[4] A.P. Meliopoulos, Power System Grounding and Transients. New York: Marcel-Dekker, 1988.

Frequency and Time Response of Power Plant Grounding System Exposed to Lightning Strike (Srete N.) 
[5] A.C. Liew and M. Darveniza, "Dynamic model of impulse characteristics of concentrated earths", Proc. Inst. Elect. Eng., vol. 121, pp. 123-135, Feb. 1974.

[6] F. Dawalibi, "Eleetromagnetie Fields Generated by Overhead and Buried Short Conductors", Part 1, Single Conductor, lEEE Transactions on Power Delivery, Vol. PWRD-I, No. 4, October 1986, pp. 105-111.

[7] F. Dawalihi, "Electromagnetic Fields Generated by Overhead and Buried Short Conductors" Part 2, Ground Networks, IEEE Transactions on Power Delivery, vol. PWRD-1, No. 4, October 1986, pp. 112-119.

[8] J. Wang, A.C. Liew, and M. Darveniza, "Extension of dynamic model of impulse behavior of concentrated grounds at high currents", IEEE Transactions on Power Delivery, vol. 20, no. 3, pp. 2160-2165, Jul. 2005.

[9] M. Ramamoorty, M.M.B. Narayanan, S. Parameswaran, and D. Mukhedkar, "Transient performance of grounding grids", IEEE Transactions on Power Delivery, vol. 4, no. 4, pp. 2053-2059, Oct. 1989.

[10] L. Zong, Y. Yin, C. Zhang, L. Zhang, "Frequency dependent characteristics of grounding system buried in multylejered earth model based on quasi-static elevtromagnetic field theory", Progres in electromagnetic research, Vol. 33, pp. 169-183, 2013.

[11] L. Grcev, "Impulse Efficiency of Ground Electrodes", IEEE Transactions on Power Delivery, Vol. 24, No. 1, 2009, pp. 441-451

[12] L. Grcev and F. Dawalibi, "An Electromagnetic Model for Transients in Grounding Systems", IEEE Transac- tions on Power Delivery, Vol. 5, No. 4, 1990, pp. 1773-1781.

[13] CDEGS 1Jser's Manual, FFTSES and HIFREQ modules, Safety Engineering Service \& Technology ltd, Sept. 2014.

[14] S. Nikolovski, S. Fortin. Frequency Domain Analysis of 110/35 kV Transformer Station Grounding System Subject to Lightning Strike, IEEE PowerTech Conferencee, Budapest, 1998, pp. BPT99-119-3

\section{BIOGRAPHIES OF AUTHORS}

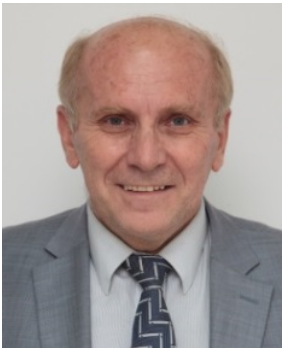

Srete Nikolovski, PhD.El.Eng. (IEEE M'1995, SM'2005) was born in Belgrade on October 1, 1954. He obtained his BSc degree (1978) and MSc degree (1989), in electrical engineering at the Faculty of Electrical Engineering, University of Belgrade and his $\mathrm{PhD}$ degree from the Faculty of Electrical and Computing Engineering, University of Zagreb, Croatia in 1993. Currently he is a Full Professor at Power Engineering Department at Faculty of Electrical Engineering, J.J. Strossmayer University in Osijek, Croatia. His main interests are power system protection, power system modeling, simulation and reliability. He has published 180 technical papers in journals and international conferences. He is a Senior Member of IEEE Reliability Society, PES Society and the member of Croatian National Committee of CIGRE

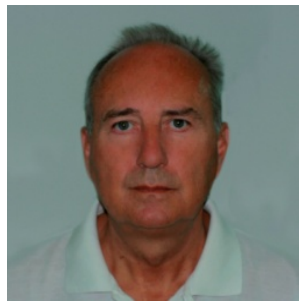

Baus Zoran, (PhD), was born in Pozarevac on June 15, 1951. He obtained his BSc degree (1975), MSc degree (1987), in electrical engineering and his $\mathrm{PhD}$ degree from the Faculty of Electrical and Computing Engineering, Zagreb, Croatia in 2004. His major fields of interest include intelligent control, fuzzy control and optimization and power system analyses. Currently he is a Full professsor at Power Engineering Department at Faculty of Electrical Engineering, J.J. Strossmayer University in Osijek, Croatia

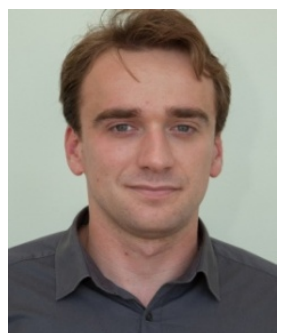

Goran Knežević, PhD, was born on September 1, 1984. He obtained his diploma degree in 2007 and his $\mathrm{PhD}$ degree in 2013 from the Faculty of Electrical Engineering, J.J. Strossmayer University in Osijek, Croatia. Presently he works as Assistant Professor at the Power System Engineering Department within the Faculty of Electrical Engineering, University in Osijek. His topics of research include electricity markets simulation, energy markets integration, power system economics and cost/benefit assessment, optimization of power system operation, power system analysis 